\title{
Comparison of geometrical, momentum and mass transfer characteristics of real foams to Kelvin cell lattices for catalyst applications
}

\author{
Francesco Lucci ${ }^{\mathrm{a}, *}$, Augusto Della Torre ${ }^{\mathrm{b}}$, Gianluca Montenegro ${ }^{\mathrm{b}}$, Rolf Kaufmann ${ }^{\mathrm{c}}$, Panayotis Dimopoulos \\ Eggenschwiler ${ }^{\mathrm{a}}$ \\ ${ }^{a}$ Automotive Powertrain Technologies, Empa, Swiss Federal Laboratories for Materials Testing and Research, Dübendorf, Switzerland \\ ${ }^{b}$ Dipartimento di Energia, Politecnico di Milano, Milano, Italy \\ ${ }^{c}$ Center for X-ray Analytics, Empa, Swiss Federal Laboratories for Materials Science and Technology, Dübendorf, Switzerland
}

\begin{abstract}
Open cell foams are considered promising catalytic substrates providing high surface area and a tortuous structure resulting in enhanced mass transfer characteristics. CFD analysis, recently, has focused in pointing structures with favourable reactivity-flow resistance characteristics. In order to reduce the geometrical complexity and computational efforts, foams have been modelled as regular (polyhedric) open cell structures. In this study a comprehensive comparison of real foams with equivalent Kelvin cell lattices is performed in CFD. Therefore 4 typical foams (two ceramic and two metallic) have been chosen. Geometric properties have been accessed with Micro-Tomography scans. Randomised Kelvin Cell lattices have been generated matching porosity and specific surface area of the scanned real foams. Geometric, momentum and mass transfer characteristics of real foams and Kelvin cell lattices are analysed with CFD. Kelvin cell lattices showed similar behaviour in respect to their real foam equivalents, had though clearly better reactivity-pressure drop trade-offs. Based on the results presented best performances as a catalyst can be expected by $3 \mathrm{D}$ printed, additive manufactured, high porosity polyhedric structures.
\end{abstract}

Keywords: ceramic and metallic foams, randomized Kelvin cell lattices, CT-scans, Masstransfer and flow simulation, Catalytic performance

\section{Introduction}

Catalyst technologies for exhaust aftertreatment for internal combustion engine applications have succeeded in reducing emissions dramatically. Cold start and low temperature emissions are though still a major concern. Catalytic acceleration of chemical reactions in the exhaust gases occurs on finely dispersed noble metal particles (Pt, $\mathrm{Pd}$ and $\mathrm{Rh}$ ), who constitute a significant cost factor. The most common substrates for modern automotive catalysts are honeycomb structures. The exhaust flow in the channels is laminar with low heat and mass transfer characteristics. For

${ }^{*}$ Corresponding author: francesco.lucci@empa.ch 
compensation, modern catalysts have far too large dimensions.

Compared to conventional honeycombs, open cell structures (foams) reach the same conversion rate but with reactors 2.5-3 times smaller [3]. Tortuous flow paths through porous structures achieve a higher chemical activity per unit of volume [1,2]. In addition Open cell structures result in higher flow uniformity which is a key factor for the pollutant conversion efficiency and for the catalyst durability $[4,5,6,7,8]$. The higher porosity and lower thermal inertia of foams are beneficial during light off [9], helping to reduce cold start emissions of vehicles. Open cell foam structures allow also more flexibility in the geometrical configuration of the reactor, since they do not restrict the lateral flow [10].

The increased mass and momentum transfer properties of foams compared to standard honeycombs result in higher conversion efficiencies but also in a higher pressure drop per unit of length [11], which, especially in the automotive field, is a crucial aspect, since it affects the global engine efficiency. Thus, to properly evaluate the reactor performance, pollutant conversion needs to be weighted by the pressure drop [1]. Experimental analysis by Giani et al.[1] and Patcas et al. [12] reported that the conversion to pressure drop trade off is higher on honeycombs compared to foams. Similar conclusion can be reached by comparing pressure drop correlations present in literature[13]. However CFD analysis by Lucci et al. $[14,15]$ suggested that the trade off is on favour of foams when their porosity is high enough.

Computational analysis has been proved useful to study flow resistance and heat transfer in open cell structures[33, $42,43,44,36,45,28,29,39]$. Despite these efforts to characterize foam transfer properties, there is no agreement for correlations that reliably predict foam performance [16], in agreement with experimental data. Edouard et al. [16] reported that no pressure drop correlation resulted consistently in good results and that standard deviations of experimental values can be as high as $100 \%$. More recently, Dietrich [17] proposed a pressure drop correlation in non dimensional form predicting most of data reported in literature within an error range of $\pm 40 \%$. A similar uncertainty is shown in a number of studies dealing with heat and mass transfer [18, 19, 20,21]. Main reasons for the ambiguities in characterizing foams are their wide range of pore sizes, different connectivities and strongly varying ligament lengths.

In literature, foams are frequently modelled as regular cell structures. These models are used for theoretical analysis $[22,23,24]$, or to derive properties which are difficult to extract experimentally, like the specific surface Area $\left(S_{v}\right)[1,25,26]$.

Current computing capabilities allow performing computational analysis of real CT foam scans [27, 28, 29, 30]. Alternatively, foams can also be accurately reconstructed with elaborate algorithms based on Voronoi tessellations [31, 32]. However, regularly structured geometries are easier to handle [33, 34, 35, 36, 37, 38, 39]. Automatic procedures can be implemented to quickly generate regular structures with prescribed properties. Moreover the geometrical 
scalability and the reduced complexity of regular structures allow more systematic analysis. In fact, while real foam structures are typically characterized by three parameters (porosity, pore diameter and specific surface), only two parameters are necessary for the regular structures since the specific surface can be obtained by precise correlations from the cell dimension and the porosity. Frequently used geometries for cell modelling are cubic cells [22, 40, 19, 23, 24] and tetrakaidecahedrons, also called Kelvin cells (KC) [25, 35, 26, 36, 41, 15].

A flow resistance comparison between Kevin Cell and foams was performed by Habisreuther et al. [35]. The authors calculated the pressure drop from a scan of an 45 PPI allumina foam with a porosity of 0.79 . In their case a randomized Kelvin Cell structure with the same properties underestimated the pressure drop compared to the foam scan. Then they show that by closing about $40 \%$ of the pores in the Kelvin cell structure similar pressures drops were achieved, they also show that by closing the structure pores they achieve similar tortuosity between the two structures.

Moreover, recent developments in additive manufacturing techniques have opened the possibility to manufacture real regular structures $[46,47]$ and potentially elevating these structures from being a model to be an actual catalytic support for commercial use. Thus, recently regular structures have also been investigated experimentally [48, 37]

In the present work open cell foams are reconstructed with randomised Kelvin cell structures and both flow resistance and mass transfer properties are analysed. At first, the micro-structure of 4 different foam samples is reconstructed by means of micro-CT scans in order to have a reliable characterization of all the geometrical properties, even those (e.g. specific surface, pore diameter) which are usually difficult to assess experimentally. Then, randomized KC structures are modelled by matching the porosity and the specific surface of the foam samples considered. Geometrical properties of the reconstructed $\mathrm{KC}$ structures are compared to the original micro-CT foam scans, to evaluate the deviations between the two 3D models. Finally, CFD investigations are performed to compare the momentum and mass transfer performances of the two kind of structures.

\section{Method}

\subsection{Micro-CT foam reconstruction}

Micro Computed Tomography has been applied for the reconstruction of the actual geometry of the samples. In a micro-CT scanner a X-ray cone beam passes through the sample and is collected by a detector; the sample is rotated providing a series of 2D projection images at different angles. A 3D voxel dataset is then reconstructed from the stack of 2D images using inverse methods. In the current case a Nikon Metrology Benchtop 160 micro-CT system was used; this uses an electron gun operating at up to $160 \mathrm{keV}$ and a metal target to generate a cone of $\mathrm{X}$-rays through bremsstrahlung; both the electron gun voltage and target metal can be altered to provide a range of spectra and penetration suitable for imaging a range of material compositions from soft biological samples to metal composites, 
including the foams used here. The sample is mounted in the beam between the target and a flat panel detector, and rotated to provide the $2 \mathrm{D}$ projection images. The exact resolution depends on the ratio between the target-sample and target-detector distances and is thus dependent on the beam angle and sample dimensions. Resolutions down to about $20 \mu m$ were achieved.

\subsection{Algorithm for the geometrical analysis}

Both real foams and KC structures are characterized in terms of porosity $\varepsilon$, specific surface $S_{v}$, pore diameter $d_{p}$ and window size $d_{w}$. These quantities are extracted from the computational mesh. While volumes and surfaces (i.e. $\varepsilon$ and $S_{v}$ ) are directly available from the computational mesh, the pore and window sizes need a statistical analysis. Thus they are computed on the basis of the opening-size approach adopted by Vogel [49]. The method is based on the fact that the foam characteristic diameters (i.e. $d_{p}$ or $d_{w}$ ) can be defined as the diameters of the largest spheres inscribed into the foam morphological characteristics of interest (i.e. pore or window) [39]. This consists of associating at every point in the space the diameter of the largest sphere which includes the point and fits completely within the void space. On this basis, the algorithm reconstructs the void space with a set of regular spherical elements characterized by different diameters. For a foam, the typical diameter distribution exhibits two distinct maxima related to the size of the pore and of the window [50]. Statistical analysis of the void size distribution allows to provide mean value and standard deviation for the cell and pore dimension. A more detailed analysis of the geometrical characterization algorithm can be found in Della Torre et al.[50].

\subsection{Kelvin cell foam reconstruction}

A full characterization of the $\mathrm{CT}$ foam scans structure is performed following the procedure summarized in section 2.2 and reported in detail by Della Torre et al.[50]. Once the main geometrical parameters of the foams are characterized, CAD-3D models of the KC structures are created consisting of a randomized structure with four $\mathrm{KCs}$ in the flow direction and two cells in the cross flow directions. Due to the high porosity and the regularity of the real foam samples used no close pore windows were considered [35]. The 3D model and the randomization of the structure are performed in such a way that they guarantee the periodicity in the cross flow directions at the domain boundaries.

The Kelvin Cell structures are build to represent as much as possible the same characteristics of the foam samples analysed. To do so, first, a KC structure is build to match each foam porosity. This was achieved by choosing the proper ratio between Kelvin Cell external pore size and strut diameter $\left(d_{s} / D_{p}\right)$ based on eq. (1) derived in Lucci et al.[14]. Then, the Kelvin Cell dimension $\left(D_{p}\right)$ is selected in order to match the specific surface area $\left(S_{v}\right)$, according to eq. (2) also derived in Lucci et al.[14]. Parameters are further corrected and iteratively fine tuned in order to guarantee 


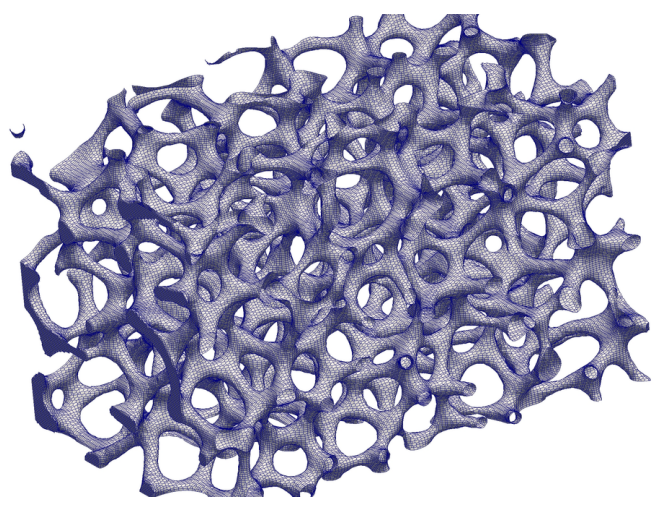

b)

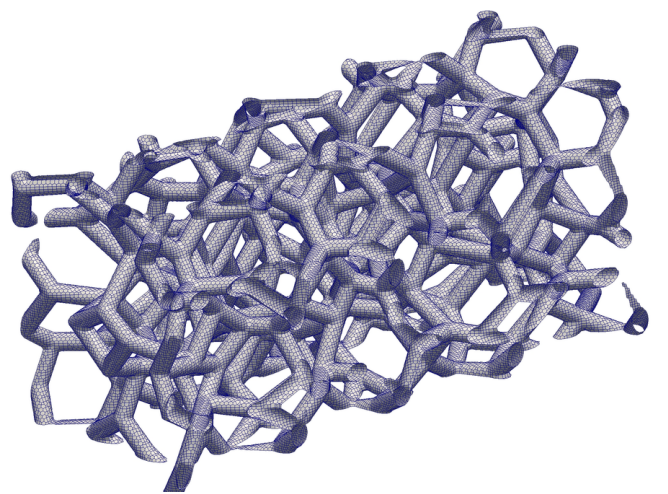

Figure 1: Sample of the final surface grid resolution: a) micro-CT of foam sample B, b) equivalent randomized Kelvin Cell lattice (B-KC).

that the values of $\varepsilon$ and $S_{v}$ between KC and foams, derived from the final computational mesh, are matched also after the randomization.

$$
\begin{aligned}
& \varepsilon=1-\frac{3 \pi}{\sqrt{2}}\left(\frac{d_{s}}{D_{p}}\right)^{2}+7.54\left(\frac{d_{s}}{D_{p}}\right)^{3} \\
& S_{v}=10.33 \frac{\sqrt{1-\varepsilon}}{D_{p}}-5.8 \frac{1-\varepsilon}{D_{p}}
\end{aligned}
$$

\subsection{Numerical modelling and assumptions}

The computational grid for both the KCs and the micro-CT foam scans is generated using the same procedure presented in [14], which employs the blockMesh and snappyHexMesh tools available in OpenFOAM.

A visualization of the computational grid is shown in Figure 1 and in Figure 2 where the grid surface resolution between a foam and a KC can be compared. All the meshes are of the order of 100000 grid elements and, to increase accuracy, they are refined at the active catalytic surface. The final meshes are made mainly by hexahedral grid elements. The remaining elements, constituting around $10 \%$ of the total are general polyhedral elements.

The global domain dimensions $\left(L_{x}, L_{y}, L_{z}\right)$ and the number of computational cells are reported in Table 1. Note that the domain dimensions between each foam and its corresponding $\mathrm{KC}$ reconstruction differ. This because the latter 
a)

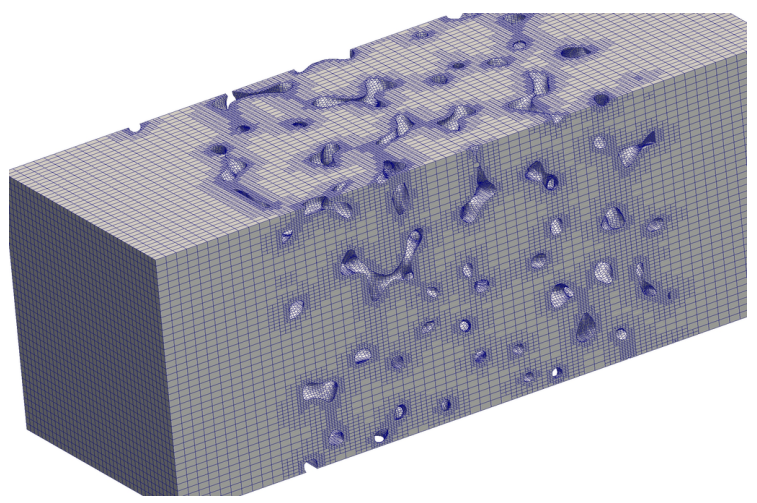

b)

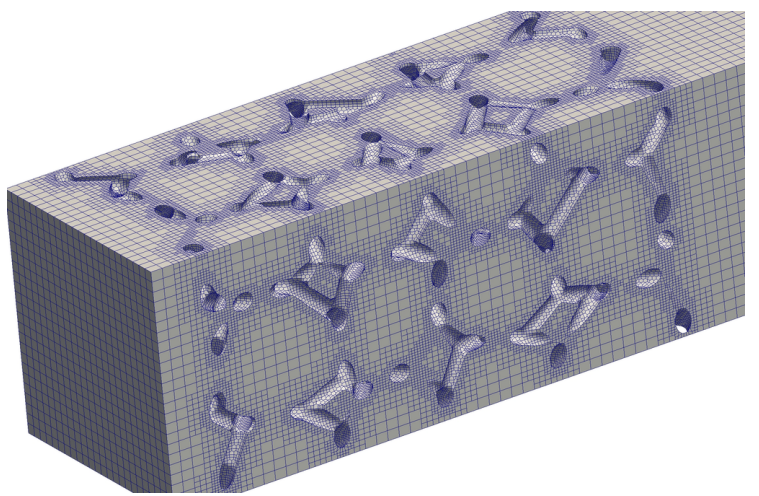

Figure 2: Sample of the final computational grid: a) foam (A) structure, b) KC (A-KC) structures.

is constructed to include only a $4 \times 2 \times 2$ array of cells.

\begin{tabular}{ccccc}
\multicolumn{5}{c}{ Table 1: Computational domains properties. } \\
\hline & case & $\begin{array}{c}\mathrm{Lx} \\
{[\mathrm{mm}]}\end{array}$ & $\begin{array}{c}\mathrm{Ly}, \mathrm{Lz} \\
{[\mathrm{mm}]}\end{array}$ & $\begin{array}{c}\text { number } \\
\text { of cells }\end{array}$ \\
\hline Foam: & $\mathrm{A}$ & 31.0 & 14.9 & 535956 \\
& $\mathrm{~B}$ & 5.0 & 1.9 & 924967 \\
& $\mathrm{C}$ & 31.0 & 6.6 & 899707 \\
Kelvin & $\mathrm{D}$ & 16.0 & 8.9 & 832814 \\
cell: & $\mathrm{A}-\mathrm{KC}$ & 35.9 & 12.0 & 412746 \\
& $\mathrm{~B}-\mathrm{KC}$ & 4.5 & 1.5 & 393249 \\
& $\mathrm{C}-\mathrm{KC}$ & 20.7 & 6.9 & 384122 \\
& $\mathrm{D}-\mathrm{KC}$ & 17.9 & 6.0 & 334071 \\
\hline
\end{tabular}

The catalyst is assumed to have reached a steady state behaviour. The SIMPLE algorithm has been implemented in a modified version of reactingFOAM, a standard OpenFOAM solver. The transport of methane $\mathrm{CH}_{4}$ in air is simulated. The Sutherland model is applied for the transport of chemical species properties and the thermal properties are extracted from JANAF tables. The methane inflow mass concentration is $X_{C H 4}=0.001$ and is assumed to have Schmidt number equal to 1 . Since simulations are performed in the transport limited regime, a constant temperature of $700 \mathrm{~K}$ is assumed at the catalyst entrance. Tests with conjugate heat transfer between the solid and the fluid show a 
difference between the maximum and minimum temperature in the solid matrix of less than 3 degrees. Thus, during production runs, a constant temperature of $750 \mathrm{~K}$ is imposed at the solid-fluid interface and no conjugate heat transfer is solved.

Gas phase reactions are neglected. Whereas infinitely fast heterogeneous reactions are modelled at the solid-fluid interface as Boundary Conditions, imposing a null concentration for the oxidized species (i.e $\mathrm{CH}_{4}$ ). Consequently the gradient of the other chemical components is corrected on the basis of the reaction stoichiometry:

$$
\frac{\partial X_{i}}{\partial \mathbf{n}}=\alpha_{i} \frac{M_{i}}{M_{C H 4}} \frac{\partial X_{C H 4}}{\partial \mathbf{n}},
$$

where $\alpha_{i}$ is the stoichiometric coefficient of specie i. At the $x^{-}$boundary are applied fixedValue inflow conditions, at the $x^{+}$boundary inletOutlet conditions and cyclic conditions are applied in the $y$ and $z$ directions. Further details on the Numerical modelling, assumptions and on the general procedures used in the present work can be found in Lucci et al.[14]. Moreover in Lucci et al.[14] the pressure drop and the mass transfer properties predicted by the present procedure are analysed and validated against a selection of the most used correlations.

\section{Results and discussion}

\subsection{Geometrical characterization}

\subsubsection{Foams}

The properties of the foam samples used for the present study (see Figure 3) as computed from the micro-CT scans are listed in Table 2. The procedure described in section 2.2, according to Della Torre et al. [50], is used to quantify the detailed geometrical properties of the foam samples. The two ceramic foams (SiC) had lower porosity $(\varepsilon=82 \%$ and $88 \%)$ while metallic foams $(\mathrm{Cu}$ and $\mathrm{Al})$ have higher porosity $(\varepsilon>90 \%)$. The commercial nomination in pores per inch (PPI), often referring to the polyurethane foams for replica manufacturing, ranges from 10 to 80 PPI. Specific surfaces of the samples have been computed in the range of $600<S_{v}<800 \mathrm{~m}^{2} / \mathrm{m}^{3}$ except for the lowest pore size foam which has a much higher surface area in the order of $S_{v}=4000 \mathrm{~m}^{2} / \mathrm{m}^{3}$. Figure 3 shows the micro-CT scans of the actual foams analysed in the CFD simulations.

Further results are included in Table 2 where the cell $\left(d_{p}\right)$ and window $\left(d_{w}\right)$ diameters, together with the respective standard deviations $\left(\sigma_{d}\right)$, have been reported. The standard deviations $\sigma_{d}$ are of the order of 10-20\% of the diameter values.

Dimensional reasoning leads to the approximation $d_{p} \approx 2 \varepsilon / S_{v}$ and $d_{w} \approx 25.4 / P P I$ (Table 2 ) for the cell and window $\left(d_{w}\right)$ diameters. $\varepsilon / S_{v}$ represents the average distance of the empty spaces from the closest catalytic surface. 

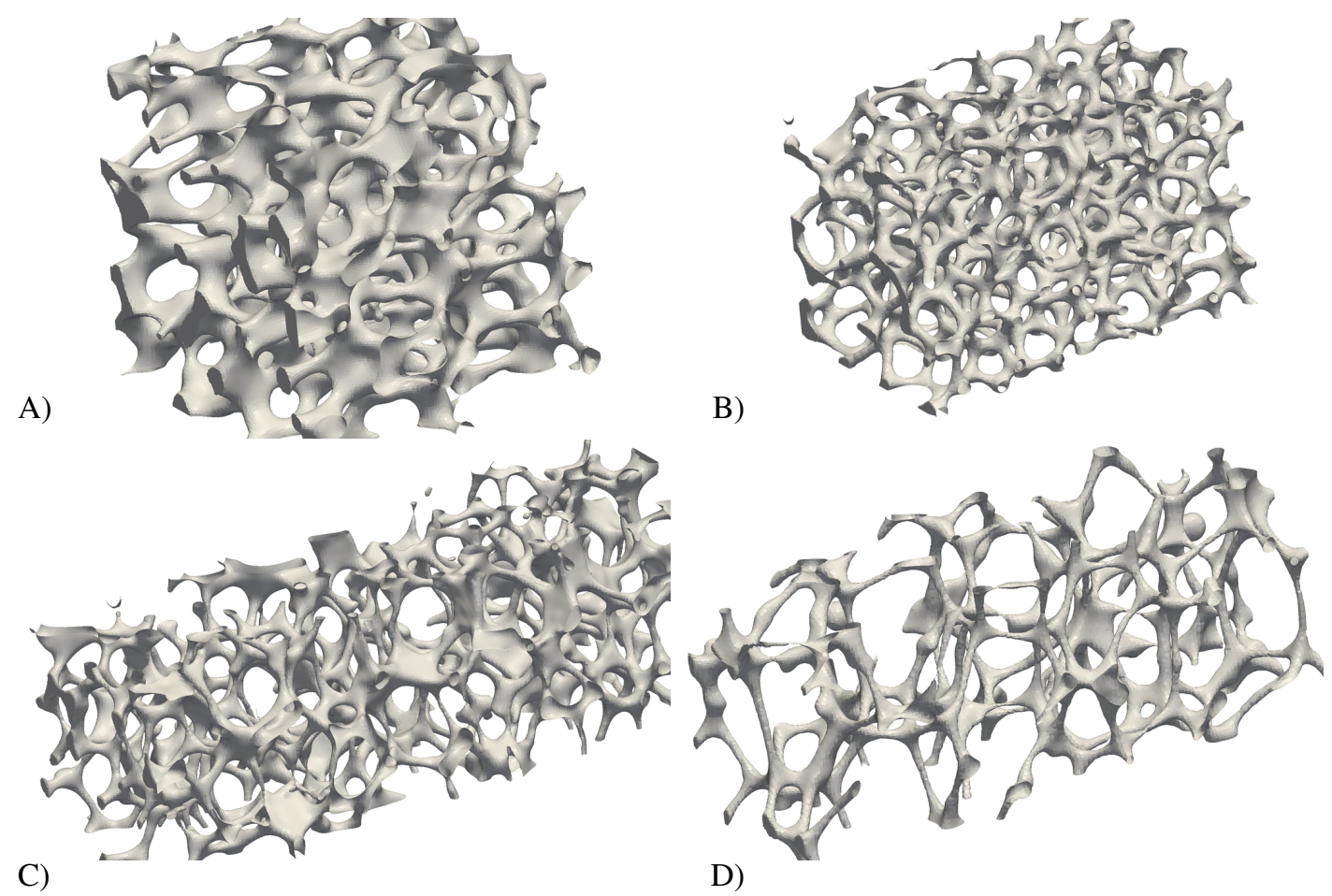

Figure 3: Micro-CT scans of the foams analysed.

\begin{tabular}{ccccccccccc}
\multicolumn{10}{c}{ Table 2: Geometric properties of foam samples computed from the micro-CT scans. } \\
\hline case & material & $\begin{array}{c}\varepsilon \\
{[-]}\end{array}$ & $\begin{array}{c}\text { PPI } \\
{[-]}\end{array}$ & $\begin{array}{c}S_{v} \\
{[1 / m]}\end{array}$ & $\begin{array}{c}2 \varepsilon / S_{v} \\
{[\mathrm{~mm}]}\end{array}$ & $\begin{array}{c}25.4 / P P I \\
{[\mathrm{~mm}]}\end{array}$ & $\begin{array}{c}d_{p} \\
{[\mathrm{~mm}]}\end{array}$ & $\begin{array}{c}\sigma_{d_{p}} \\
{[\mathrm{~mm}]}\end{array}$ & $\begin{array}{c}d_{w} \\
{[\mathrm{~mm}]}\end{array}$ & $\begin{array}{c}\sigma_{d_{w}} \\
{[\mathrm{~mm}]}\end{array}$ \\
\hline $\mathrm{A}$ & $\mathrm{SiC}$ & 0.827 & 10 & 590 & 2.8 & 2.54 & 3.8 & 0.56 & 1.9 & 0.54 \\
$\mathrm{~B}$ & $\mathrm{SiC}$ & 0.877 & 80 & 4120 & 0.42 & 0.32 & 0.51 & 0.047 & 0.27 & 0.058 \\
$\mathrm{C}$ & $\mathrm{Cu}$ & 0.897 & 20 & 820 & 2.2 & 1.3 & 2.2 & 0.29 & 1.3 & 0.23 \\
$\mathrm{D}$ & $\mathrm{Al}$ & 0.955 & 40 & 690 & 2.7 & 0.64 & 2.0 & 0.33 & 1.1 & 0.27 \\
\hline
\end{tabular}

25.4/PPI uses the PPI definition (Pores Per Inch) to predict the $d_{w}$. A comparison of the values obtained and the data evaluated from the CT scans leads to correct predictions of both $d_{p}$ and $d_{w}$ for foam C, and acceptable predictions for foam B. However in foams A and D an over- or under- estimation of the order of $30 \%$ was obtained.

\subsubsection{Kelvin Cells}

The procedures described in 2.1 are used to obtain the $\mathrm{KC}$ structures equivalent to the scanned real foams. A schematic of the front view of a $\mathrm{KC}$ is presented in Figure 4. The main characteristic lengths sketched in Figure 4 and the geometrical properties of the building KCs are summarized in Table 3, note that here the cell diameter $d_{p}^{K C}$ refers to the size of the $\mathrm{KC}$ used as a building block for the $\mathrm{KC}$ structure before the randomization process. It can be seen that, in order to match the porosity and the specific surface of the CT foam scan, the value of the cell diameter $d_{p}^{K C}$ needs to be chosen about 30\% higher than the corresponding foam pore diameter. Deviations between the foam pore 


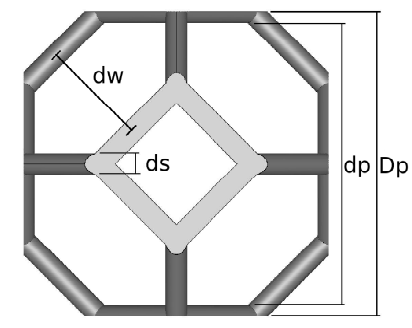

Figure 4: Front view of $\mathrm{KC}$ with characteristic dimensions.

diameters and the cell diameters $d_{p}^{K C}$ are reported in Table 3 as $\Delta d_{p}^{K C}$.

\begin{tabular}{|c|c|c|c|c|c|}
\hline case & $\varepsilon$ & $\begin{array}{c}S_{v} \\
{\left[m^{2} / m^{3}\right]}\end{array}$ & $\begin{array}{l}d_{p}^{K C} \\
{[\mathrm{~mm}]}\end{array}$ & $\begin{array}{l}d_{s}^{K C} \\
{[\mathrm{~mm}]}\end{array}$ & $\begin{array}{c}\Delta d_{p}^{K C} \\
{[\%]}\end{array}$ \\
\hline A-KC & 0.828 & 590 & 4.958 & 1.022 & 30.4 \\
\hline $\mathrm{B}-\mathrm{KC}$ & 0.878 & 4150 & 0.642 & 0.106 & 25.8 \\
\hline C-KC & 0.897 & 840 & 3.007 & 0.442 & 36.6 \\
\hline $\mathrm{D}-\mathrm{KC}$ & 0.956 & 680 & 2.743 & 0.247 & 37.1 \\
\hline
\end{tabular}

After the randomization KC structures are analysed with the same technique (sec. 2.2) used for the foams. Results are presented in Table 4 . The $d_{p}$ computed is always about $15 \%$ smaller than the non randomized $d_{p}^{K C}$ of the regular structure presented in Table 3. This is a consequence of the randomization that deforms the internal pores reducing the size of the maximum sphere fitting in them. Thus the randomization reduces the deviation between the cell diameter $d_{p}$ of the KC structure and of the real foams from $30 \%$ to a maximum of $18 \%$.

Percentage deviations of the properties between the foams and their KC reconstruction are reported in Table 5. It can be seen that the procedure here presented is able to guarantee very good agreement in terms of porosity $(\Delta \varepsilon \gtrless 0.1 \%)$, specific surface $\left(\Delta S_{v} \gtrless 2.5 \%\right)$ and window diameter $\left(\Delta d_{w} \gtrless 4 \%\right)$.

A more detailed analysis is presented in Figure 5 where the distributions of typical dimensions of the voids of each foam and its $\mathrm{KC}$ reconstruction are plotted. The peak at lower values refers to the windows' diameter while the peak on the right refers to the pore or cell diameter. The diameter distributions of the foams and of the corresponding KC lattices are similar, suggesting that the two structures have similar statistical characteristics.

However few differences can be noticed. First, for the $\mathrm{KC}$, the pore diameter $d_{p}$ peak is shifted to the right resulting, as we have seen, in an higher $d_{p}$ average value. Second, for the KC structures the window diameter $d_{w}$ is characterized by a bimodal distribution, evident from the presence of a secondary peak at lower values. So, although its final average coincides with the foam value, it presents a higher standard deviation $\left(\sigma_{d_{w}}\right)$, as reported in Table 4. This bimodal distribution is clearly caused by the geometrical structures of the Kelvin Cells, where windows can have 
a square form with diameter of $d_{w} \approx d_{p} / 2$ or an hexahedral form with diameter $d_{w} \approx d_{p} / \sqrt{2}$ (see Figure 4).

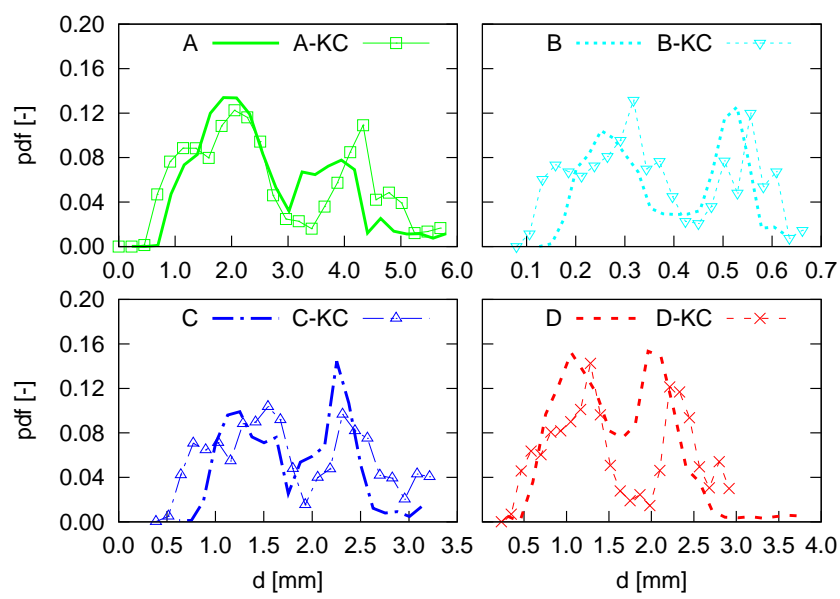

Figure 5: Diameter distributions for the four sample foams and the corresponding KC structures.

Table 4: Diameter and Standard deviation of randomised KC structures. From algorithm in sec.2.2.

\begin{tabular}{ccccc}
\hline case & $d_{p}[\mathrm{~mm}]$ & $\sigma_{d_{p}}[\mathrm{~mm}]$ & $d_{w}[\mathrm{~mm}]$ & $\sigma_{d_{w}}[\mathrm{~mm}]$ \\
\hline A-KC & 4.3 & 0.55 & 1.8 & 0.65 \\
B-KC & 0.55 & 0.047 & 0.28 & 0.090 \\
C-KC & 2.5 & 0.36 & 1.3 & 0.38 \\
D-KC & 2.4 & 0.25 & 1.1 & 0.35 \\
\hline
\end{tabular}

Table 5: Percentage properties deviations between randomised KC and foams.

\begin{tabular}{ccccc}
\hline case & $\Delta \varepsilon[\%]$ & $\Delta S_{v}[\%]$ & $\Delta d_{p}[\%]$ & $\Delta d_{w}[\%]$ \\
\hline A & 0.1 & 0.0 & 15 & -3.9 \\
B & 0.1 & 0.7 & 8.0 & 0.8 \\
C & 0.0 & 2.4 & 14 & -0.3 \\
D & 0.1 & -1.5 & 18 & 1.3 \\
\hline
\end{tabular}

\subsection{Transport analysis}

\section{Pressure Drop}

The pressure drop per unit length of the flow through real foams and the $\mathrm{KC}$ lattices is plotted in Figure 6 . Real foam pressure drops are plotted with lines, while the ones for randomized KC lattices are plotted with symbols with the same color of the corresponding foam line. As expected pressure drops of the open cell structures have an exponential increase with increasing velocity. As shown by [14] (see eq.13 in [14]), structures with the highest specific surface area lead to the highest pressure drop.

The $\mathrm{KC}$ cases are able to reproduce quantitatively the pressure drop of foams. The percentage deviation between foams and the relative $\mathrm{KC}$ for the pressure and mass transport statistics are plotted in Figure 9. The maximum pressure 


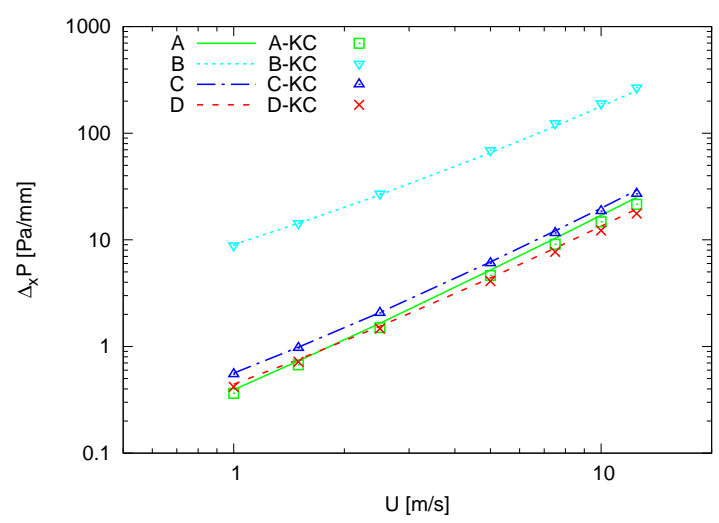

Figure 6: Pressure drop per unit length in $\mathrm{Pa} / \mathrm{mm}$ versus surface velocity.

drop deviation between the two structures is less than $15 \%$ even if our simulations span a range of pressure drops of 3 orders of magnitude. In all cases, KC lattices result in lower pressure drop, apart from the smallest pore diameter (i.e. case $\mathrm{B}$ ), where at high velocities the pressure drop of the $\mathrm{KC}$ lattice is slightly higher.

\section{Mass transfer coefficients}

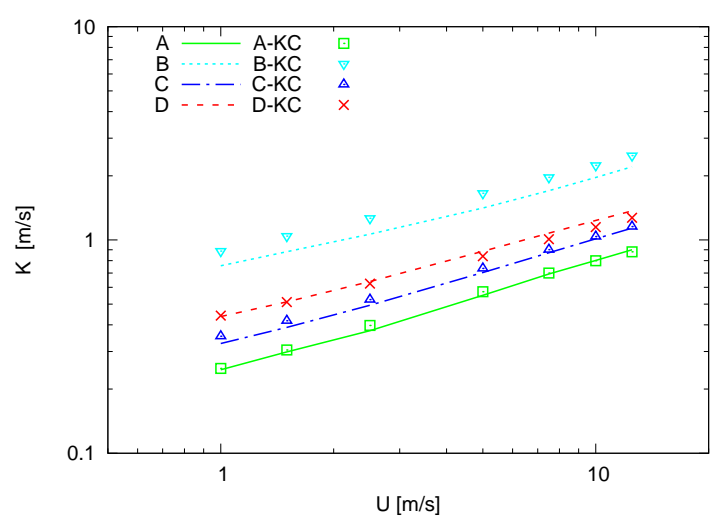

Figure 7: Mass transfer coefficient (Eq.4) versus inflow velocity.

The mass transfer coefficient $K$ for all cases is plotted in Figure 7. The parameter $K$ can be expressed as[51]:

$$
K=-\frac{\ln (1-\eta)}{S_{v} L_{R} / U}
$$

where $L_{R}$ is the length of the catalyst and $\eta$ the conversion rate. The conversion term is evaluated from the inflow and outflow average methane molar fractions : $(1-\eta)=Y_{\mathrm{CH}_{4}}^{\text {in }} / Y_{\mathrm{CH}_{4}}^{\text {out }}$.

The maximum deviation between foam and $\mathrm{KC}$ is experienced by lowest pore size(i.e. case $\mathrm{B}$ ) where $\mathrm{KC}$ mass transfer is approximately $15 \%$ higher, with a maximum deviation of less than $20 \%$ (Figure 9). The other cases have lower deviations of about $5 \%$. 


\section{Pressure drop and mass transfer trade off}

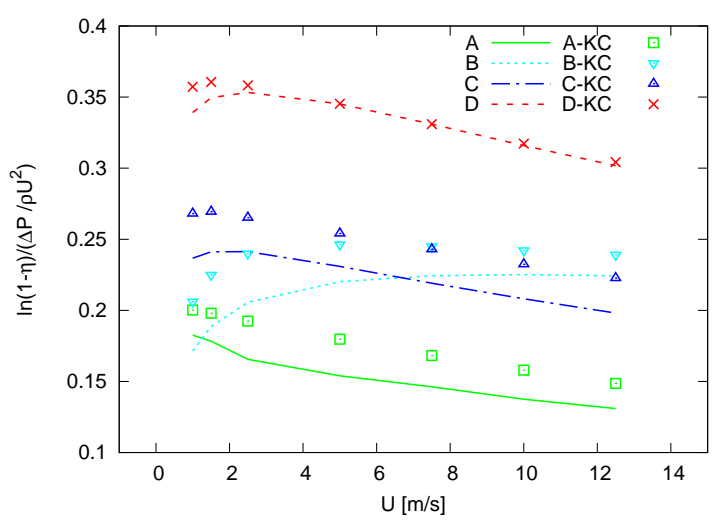

Figure 8: Performance coefficient $I$ (Eq.5) versus inflow velocity.

In order to evaluate the efficiency of the catalytic conversion with respect to pressure drop, Giani et al. [1] defined a dimensionless parameter $I$ as

$$
I=\frac{-\ln (1-\eta)}{\Delta P / \rho U^{2}} .
$$

At the same operational velocity $U$, more efficient structures have higher index $I$ since they can deliver higher conversion $(\eta)$ for the same pressure drop $(\Delta P)$.

The index $I$ is plotted in Figure 8. It is clear that $\mathrm{KC}$ structures outperform foams by approximately $15-10 \%$. Best performance is reached by the foams/KC lattices with the highest porosity. This consistent with our previous results [14]. Interestingly, for the highest porosity case, at low velocities the deviation between foams and KC lattices is below 5\%. At high velocities the differences decrease and the agreement between foams and KCs is remarkable.
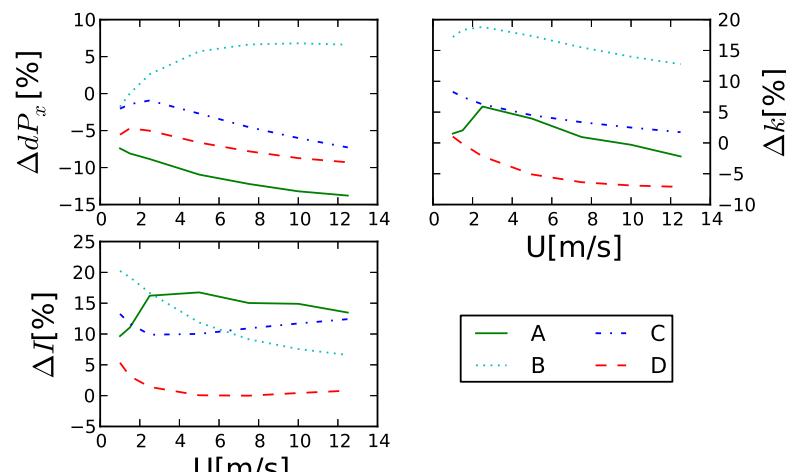

Figure 9: Percentage deviations, between foam structures and their KC representations, for the pressure drop, the mass transfer coefficient and the performance index.

Best agreement between the real foam and the KC Lattice model is reached also for case $\mathrm{D}$. This is the foam/KC 
lattice with the highest porosity. The good agreement can be explained by the fact that in this case foam struts are similar to the long cylindrical struts of the $\mathrm{KC}$ model, no closed pores are present and there is little material accumulation at the cell nodes (see Figure 3D ). Thus, the KC model better reproduces the CT foam scan. Note, also, that for this case both the pressure drop and the mass transfer coefficient of the KC model are lower by about 5-10\% (Figure 9), indicating that a more uniform structure of the $\mathrm{KC}$ model is still offering a slightly smaller resistance to the flow. However, given the small deviations in the performance index $I$, the balance between momentum and mass transfer follows the correct laws and it is well represented.

\section{Non-dimensional correlations}

Given the geometrical complexity of foams non-dimensional correlations are valuable tools to analyse their performances. Recently, Dietrich [17] proposed a non-dimensional pressure drop correlation predicting most of the 2500 analysed literature data points within an error range of $\pm 40 \%$ :

$$
H g=110 R e+1.45 R e^{2} .
$$

The dimensionless pressure drop (Hagen Number) is defined as :

$$
H g=\frac{\Delta P}{\Delta x} \cdot \frac{D_{h}^{3}}{\rho v^{2}},
$$

and the Reynolds number is :

$$
R e=\frac{u \cdot D_{h}}{\varepsilon \cdot v},
$$

where here the hydraulic diameter, $D_{h}$, is defined from the specific surface and the porosity:

$$
D_{h}=4 \cdot \frac{\varepsilon}{S_{v}} .
$$

In Figure 10 we compare our results of foams and their $\mathrm{KC}$ reconstructions to the correlation proposed by Dietrich (Eq. 6). We see that most of our results are well within the $40 \%$ error band indicated by Dietrich [17]. Only the sample D and its reconstruction D-KC at low velocities are touching and slightly passing the $40 \%$ limit. However, sample D is an Aluminium foam (Table 2). This is consistent with the data reported by [17] where some aluminium foams are experiencing a slightly higher deviation than $40 \%$.

A non-dimensional correlation to visualize the trade off between pressure drop and mass transfer is the Lévêrque analogy presented by Martin [52] for heat exchangers. Incera Garrido et al. [20] verified that the analogy holds also 


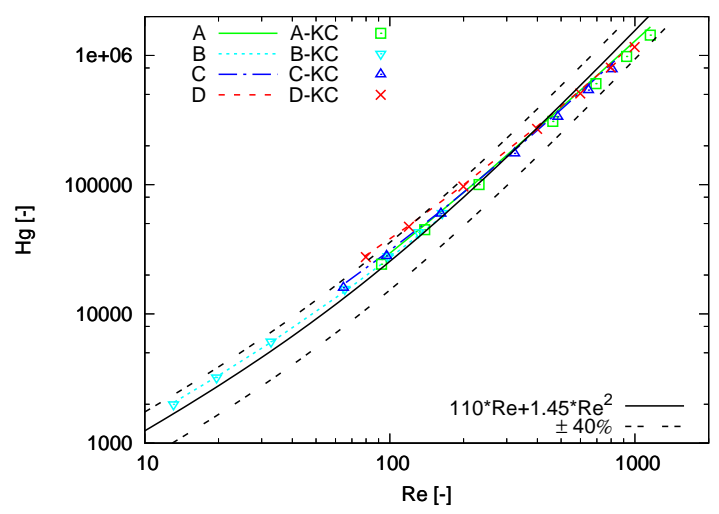

Figure 10: Non-dimensional pressure drop (Hagen number, $\mathrm{Hg}$ ) versus Reynolds Number, Re.

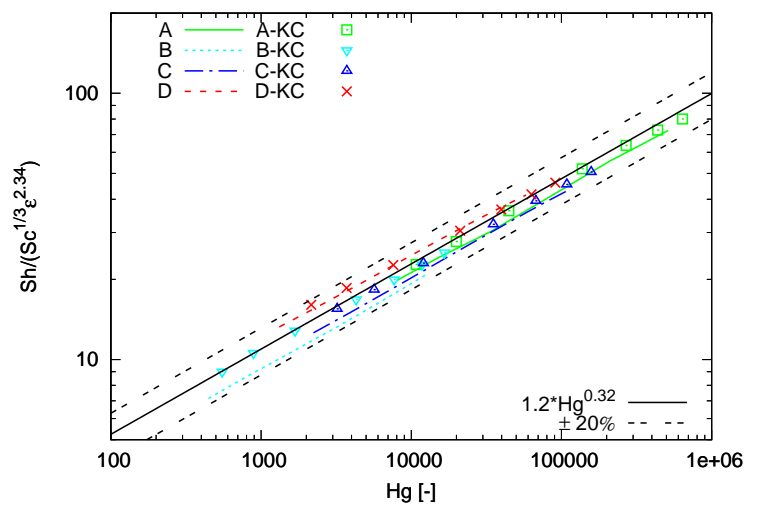

Figure 11: Shervood number, Sh, versus Hagen number, Hg.

for catalytic foams and presented a specific correlation:

$$
S h_{I G}=0.62\left(\frac{D_{p}}{0.001 m}\right)^{0.48} \cdot \varepsilon^{2.34} \cdot H g^{0.31} \cdot S c^{1 / 3}
$$

Here $\mathrm{Sh}$ is the Sherwood number $\left(S h=K D_{P} / D_{\text {air }}\right)$, Sc is the Schmidt number and $\mathrm{Hg}$ is the Hagen number. Note that differently from [17], Incera Garrido et al. [20] use directly the pore diameter $D_{p}$ as hydraulic diameter.

The Sherwood number corrected by the porosity $\left(S h / \varepsilon^{2.34} S c^{1 / 3}\right)$ is plotted versus the Hagen number in Figure 11. Both foams and $\mathrm{KC}$ structures scale with $1.2 \mathrm{Hg}^{0.32}$ but with a deviation of approximately $20 \%$. The correlation described in eq. (10) and presented by Incera Garrido et al. [20] presents a pre factor of 0.62 which is significantly lower than the one plotted in Figure 11. This is a consequence of the different nature of the Sherwood numbers between the two studies. Due to the assumption in the present study that all the reactants instantaneously react as soon they reach the catalytic surface (i.e. $X_{C H 4}^{\text {surf }}=0$ ), Sh represents the external mass transport limit, or the maximum transport from the bulk of the flow to the catalytic surfaces. In experimental studies, Sh includes also the chemical and diffusion resistance inside the washcoat. In particular, it is known that for open cell foam geometries the washcoat 
diffusion can reduce up to $40 \%$ of the effective reaction rate [53], hence the lower pre factor.

\section{Conclusions}

Micro-CT scans of four different ceramic and metallic open cell foams have been performed. Geometrical properties of the foams have been extracted from the CT scans with high accuracy. Porosities were between $(82<\varepsilon<96 \%)$ and specific surface areas between $590<S_{v}<4120[1 / \mathrm{m}]$, these values being suitable for automotive applications. The equivalent Kelvin cell lattices have been modelled by matching the porosity and the surface area of the four real sample foams. Notably this resulted in regularly stacked KC lattices with approximately $30 \%$ higher pore diameters, after randomization the $\mathrm{KC}$ lattices had only $15 \%$ higher pore diameters. CFD simulations were performed to investigate their momentum and mass transfer properties through real foams and $\mathrm{KC}$ lattices, the results are consistent with published correlations.

The main conclusions and achievements of the work are:

- Pressure drops through $\mathrm{KC}$ lattices are generally lower than through the equivalent real foam, apart from the foam with the lowest pore diameter.

- Mass transfer coefficients through $\mathrm{KC}$ lattices are generally higher than through the equivalent real foam, apart from the foam with the highest porosity.

- The KC lattices show always better trade off between mass transfer and pressure drop in respect to the equivalent real foam. For the low porosity structures the gain of $\mathrm{KC}$ lattices in performance index $I$ is about $15 \%$. For the highest porosity $(\varepsilon=95 \%)$ the gain is lower.

The results demonstrate the substantial advantages in terms of reactivity per pressure drop of catalysts based on KC lattices. New additive manufacturing technologies may deliver soon samples for experimental validation.

\section{Acknowledgments}

The authors gratefully aknowledge financial support from the Swiss National Science Foundation (SNF) for the project "Cases: Catalyst Substrates from Engineered Structures for state of the art powertrains: High pollutant conversion (including cold-start) with low precious metal requirements", Project No 20PC21_161571/1 and from the Italian Ministry of Education, University and Research, Rome (MIUR, Progetti di Ricerca Scientifica di Rilevante Interesse Nazionale, prot. 2010XFT2BB), within the project IFOAMS: "Intensification of Catalytic Processes for Clean Energy, Low-Emission Transport and Sustainable Chemistry using Open-Cell Foams as Novel Advanced Structured Materials". 


\section{List of Notations}

$\eta \quad$ conversion efficiency $\eta=\frac{C_{i n}-C_{o u t}}{C_{\text {in }}}[-]$

$v \quad$ kinematic viscosity $\left[\mathrm{m}^{2} / \mathrm{s}\right]$

$\sigma_{x} \quad$ standard deviation of quantity $\mathrm{x}$

$d_{p}^{K C} \quad$ pore diameter of Kelvin Cell structures before randomization [m]

$D_{h} \quad$ hydraulic diameter $[\mathrm{m}]$

$D_{p} \quad$ external pore or cell diameter $D_{P}=d_{p}+d_{s}[\mathrm{~m}]$

$d_{p} \quad$ internal pore diameter of foam or cell diameter of randomized $\mathrm{KC}$ structures [mm]

$d_{w} \quad$ window diameter of foam or randomized $\mathrm{KC}$ structures [mm]

$D_{\text {air }} \quad$ air Diffusivity $\left[m^{2} / s\right]$

$H g \quad$ Hagen number $H g=\frac{\Delta P}{\Delta x} \cdot \frac{D_{h}^{3}}{\rho v^{2}}[-]$

$K \quad$ mass transfer coefficient $K=-\frac{\ln (1-\eta)}{S_{v} L_{R} / U}[\mathrm{~m} / \mathrm{s}]$

$K C \quad$ Kelvin Cell

$L_{R} \quad$ reactor length $[\mathrm{m}]$

$L_{x, y, z} \quad$ domain dimensions [m]

PPI Pores Per Inch

Re Reynolds number $R e=\frac{D_{h} u}{\varepsilon v}[-]$

$S h \quad$ Sherwood number $S h=K D_{P} / D_{\text {air }}[-]$

$U \quad$ inflow velocity $[\mathrm{m} / \mathrm{s}]$

$Y_{X} \quad$ mass fraction of specie $X[-]$

$\varepsilon \quad$ porosity [-]

$S_{v} \quad$ specific surface area $\left[\mathrm{m}^{2} / \mathrm{m}^{3}\right]$

CT X-Ray Computer Tomography 


\section{References}

[1] L. Giani, G. Groppi, E. Tronconi, Mass-transfer characterization of metallic foams as supports for structured catalysts, Industrial and Engineering Chemistry Research 44 (14) (2005) 4993-5002.

[2] K. Pangarkar, T. Schildhauer, J. Van Ommen, J. Nijenhuis, F. Kapteijn, J. Moulijn, Structured packings for multiphase catalytic reactors, Industrial and Engineering Chemistry Research 47 (10) (2008) 3720-3751.

[3] C. Bach, P. Dimopoulos Eggenschwiler, Ceramic foam catalyst substrates for diesel oxidation catalysts: Pollutant conversion and operational issues, SAE Technical Papers (2011-24-0179).

[4] G. Gaiser, J. Oesterle, J. Braun, P. Zacke, The progressive spin inlet - Homogeneous flow distributions under stringent conditions, SAE Technical Papers.

[5] A. P. Martin, N. S. Will, A. Bordet, P. Cornet, C. Gondoin, X. Mouton, Effect of flow distribution on emissions performance of catalytic converters, SAE Technical Papers.

[6] K. Zygourakis, Transient operation of monolith catalytic converters: a two-dimensional reactor model and the effects of radially nonuniform flow distributions, Chemical Engineering Science 44 (9) (1989) 2075-2086.

[7] P. Dimopoulos Eggenschwiler, D. Tsinoglou, J. Seyfert, C. Bach, U. Vogt, M. Gorbar, Ceramic foam substrates for automotive catalyst applications: Fluid mechanic analysis, Experiments in Fluids 47 (2) (2009) 209-222.

[8] P. Parthasarathy, P. Habisreuther, N. Zarzalis, Evaluation of longitudinal dispersion coefficient in open-cell foams using transient direct pore level simulation, Chemical Engineering Science 90 (2013) 242-249. doi:10.1016/j.ces.2012.12.041.

[9] J. von Rickenbach, F. Lucci, C. Narayanan, P. D. Eggenschwiler, D. Poulikakos, Pore scale modeling of cold-start emissions in foam based catalytic reactors, Chemical Engineering Sciencedoi:10.1016/j.ces.2015.08.020.

[10] G. C. Koltsakis, D. K. Katsaounis, Z. C. Samaras, D. Naumann, S. Saberi, A. Böhm, I. Markomanolakis, Development of metal foam based aftertreatment system on a diesel passenger car, SAE Technical Papers (2008-01-0619).

[11] M. Twigg, J. Richardson, Fundamentals and applications of structured ceramic foam catalysts, Industrial and Engineering Chemistry Research 46 (12) (2007) 4166-4177.

[12] F. Patcas, G. Garrido, B. Kraushaar-Czarnetzki, CO oxidation over structured carriers: A comparison of ceramic foams, honeycombs and beads, Chemical Engineering Science 62 (15) (2007) 3984-3990.

[13] S. Lopatin, P. Mikenin, D. Pisarev, D. Baranov, S. Zazhigalov, A. Zagoruiko, Pressure drop and mass transfer in the structured cartridges with fiber-glass catalyst, Chemical Engineering Journal 282 (2015) 58-65. doi:10.1016/j.cej.2015.02.026.

[14] F. Lucci, A. Della Torre, J. von Rickenbach, G. Montenegro, D. Poulikakos, P. Dimopoulos Eggenschwiler, Performance of randomized Kelvin cell structures as catalytic substrates: Mass-transfer based analysis, Chemical Engineering Science 112 (2014) $143-151$.

[15] F. Lucci, A. Della Torre, G. Montenegro, P. Dimopoulos Eggenschwiler, On the catalytic performance of open cell structures versus honeycombs, Chemical Engineering Journal 264 (2015) 514-521.

[16] D. Edouard, M. Lacroix, C. Pham, M. Mbodji, C. Pham-Huu, Experimental measurements and multiphase flow models in solid SiC foam beds, AIChE Journal 54 (11) (2008) 2823-2832.

[17] B. Dietrich, Pressure drop correlation for ceramic and metal sponges, Chemical Engineering Science 74 (2012) $192-199$. doi:10.1016/j.ces.2012.02.047.

[18] J. Richardson, D. Remue, J.-K. Hung, Properties of ceramic foam catalyst supports: Mass and heat transfer, Applied Catalysis A: General 250 (2) (2003) 319-329.

[19] L. Giani, G. Groppi, E. Tronconi, Heat transfer characterization of metallic foams, Industrial and Engineering Chemistry Research 44 (24) (2005) 9078-9085. 
[20] G. Incera Garrido, F. Patcas, S. Lang, B. Kraushaar-Czarnetzki, Mass transfer and pressure drop in ceramic foams: A description for different pore sizes and porosities, Chemical Engineering Science 63 (21) (2008) 5202-5217.

[21] T. Huu, M. Lacroix, C. Pham Huu, D. Schweich, D. Edouard, Towards a more realistic modeling of solid foam: Use of the pentagonal dodecahedron geometry, Chemical Engineering Science 64 (24) (2009) 5131-5142.

[22] P. Du Plessis, A. Montillet, J. Comiti, J. Legrand, Pressure drop prediction for flow through high porosity metallic foams, Chemical Engineering Science 49 (21) (1994) 3545-3553.

[23] J. Fourie, J. Du Plessis, Pressure drop modelling in cellular metallic foams, Chemical Engineering Science 57 (14) (2002) $2781-2789$.

[24] J. Ahmed, C. Pham-Huu, D. Edouard, A predictive model based on tortuosity for pressure drop estimation in 'slim' and 'fat' foams, Chemical Engineering Science 66 (20) (2011) 4771-4779.

[25] J. Richardson, Y. Peng, D. Remue, Properties of ceramic foam catalyst supports: Pressure drop, Applied Catalysis A: General 204 (1) (2000) $19-32$.

[26] A. Inayat, H. Freund, T. Zeiser, W. Schwieger, Determining the specific surface area of ceramic foams: The tetrakaidecahedra model revisited, Chemical Engineering Science 66 (6) (2011) 1179-1188.

[27] E. Bianchi, T. Heidig, C. G. Visconti, G. Groppi, H. Freund, E. Tronconi, An appraisal of the heat transfer properties of metallic open-cell foams for strongly exo-/endo-thermic catalytic processes in tubular reactors, Chemical Engineering Journal 198-199 (2012) 512-528.

[28] A. Della Torre, G. Montenegro, G. Tabor, M. Wears, CFD characterization of flow regimes inside open cell foam substrates, International Journal of Heat and Fluid Flow (0) (2014) -. doi:10.1016/j.ijheatfluidflow.2014.05.005.

[29] A. Diani, K. Bodla, L. Rossetto, S. Garimella, Numerical investigation of pressure drop and heat transfer through reconstructed metal foams and comparison against experiments, International Journal of Heat and Mass Transfer 88 (2015) 508-515.

[30] W. Regulski, J. Szumbarski, L. Laniewski-Wollk, K. Gumowski, J. Skibinski, M. Wichrowski, T. Wejrzanowski, Pressure drop in flow across ceramic foams-A numerical and experimental study, Chemical Engineering Science 137 (2015) 320-337. doi:10.1016/j.ces.2015.06.043.

[31] G. D. Wehinger, H. Heitmann, M. Kraume, An artificial structure modeler for 3D \{CFD $\}$ simulations of catalytic foams, Chemical Engineering Journal 284 (2016) 543-556.

[32] T. Wejrzanowski, J. Skibinski, J. Szumbarski, K. Kurzydlowski, Structure of foams modeled by Laguerre-Voronoi tessellations, Computational Materials Science 67 (2013) 216-221. doi:10.1016/j.commatsci.2012.08.046

[33] K. Boomsma, D. Poulikakos, Y. Ventikos, Simulations of flow through open cell metal foams using an idealized periodic cell structure, International Journal of Heat and Fluid Flow 24 (6) (2003) 825-834.

[34] S. Krishnan, J. Murthy, S. Garimella, Direct simulation of transport in open-cell metal foam, Journal of Heat Transfer 128 (8) (2006) $793-799$.

[35] P. Habisreuther, N. Djordjevic, N. Zarzalis, Statistical distribution of residence time and tortuosity of flow through open-cell foams, Chemical Engineering Science 64 (23) (2009) 4943-4954.

[36] M. Bai, J. Chung, Analytical and numerical prediction of heat transfer and pressure drop in open-cell metal foams, International Journal of Thermal Sciences 50 (6) (2011) 869-880.

[37] A. Zenklusen, S. Walser, P. Rudolf von Rohr, Influence of ligament shape and thickness on vortex shedding in highly porous structures, Chemical Engineering Science 129 (2015) 1-8.

[38] A. Della Torre, F. Lucci, G. Montenegro, A. Onorati, P. Dimopoulos Eggenschwiler, E. Tronconi, G. Groppi, CFD modeling of catalytic reactions in open-cell foam substrates, Computers and Chemical Engineering 92 (2016) 55-63. doi:10.1016/j.compchemeng.2016.04.031.

[39] G. Ambrosio, N. Bianco, W. K. Chiu, M. Iasiello, V. Naso, M. Oliviero, The effect of open-cell metal foams strut shape on convection heat transfer and pressure drop, Applied Thermal Engineering 103 (2016) 333-343. doi:10.1016/j.applthermaleng.2016.04.085.

[40] A. G. Evans, J. W. Hutchinson, M. F. Ashby, Cellular metals, Current Opinion in Solid State and Materials Science 3 (3) (1998) $288-303$. 
[41] J. von Rickenbach, F. Lucci, C. Narayanan, P. D. Eggenschwiler, D. Poulikakos, Multi-scale modelling of mass transfer limited heterogeneous reactions in open cell foams, International Journal of Heat and Mass Transfer 75 (0) (2014) $337-346$. doi:10.1016/j.ijheatmasstransfer.2014.03.060.

[42] S. Krishnan, S. Garimella, J. Murthy, Simulation of thermal transport in open-cell metal foams: Effect of periodic unit-cell structure, Journal of Heat Transfer 130 (2). doi:10.1115/1.2789718.

[43] A. Kopanidis, A. Theodorakakos, E. Gavaises, D. Bouris, 3D numerical simulation of flow and conjugate heat transfer through a pore scale model of high porosity open cell metal foam, International Journal of Heat and Mass Transfer 53 (11-12) (2010) 2539-2550.

[44] Z. Wu, C. Caliot, G. Flamant, Z. Wang, Numerical simulation of convective heat transfer between air flow and ceramic foams to optimise volumetric solar air receiver performances, International Journal of Heat and Mass Transfer 54 (7-8) (2011) 1527-1537.

[45] L. Ferrari, M. Barbato, A. Ortona, C. D’Angelo, Convective heat transfer in cellular ceramic: A 3D numerical solution, International Conference on Heat Transfer, Fluid Mechanics and Thermodynamics.

[46] L. Murr, S. Gaytan, F. Medina, E. Martinez, J. Martinez, D. Hernandez, B. Machado, D. Ramirez, R. Wicker, Characterization of Ti-6Al-4V open cellular foams fabricated by additive manufacturing using electron beam melting, Materials Science and Engineering: A 527 (7-8) (2010) 1861-1868. doi:10.1016/j.msea.2009.11.015.

[47] A. Ortona, C. D’Angelo, S. Gianella, D. Gaia, Cellular ceramics produced by rapid prototyping and replication, Materials Letters 80 (2012) 95-98.

[48] A. Inayat, J. Schwerdtfeger, H. Freund, C. Körner, R. Singer, W. Schwieger, Periodic open-cell foams: Pressure drop measurements and modeling of an ideal tetrakaidecahedra packing, Chemical Engineering Science 66 (12) (2011) 2758-2763.

[49] H. J. Vogel, Morphological determination of pore connectivity as a function of pore size using serial sections, European Journal of Soil Science 48 (1997) 365-377.

[50] A. Della Torre, G. Montenegro, G. Tabor, M. Wears, CFD characterization of flow regimes inside open cell foam substrates, International Journal of Heat and Fluid Flow 50 (0) (2014) 72-82.

[51] G. Groppi, L. Giani, E. Tronconi, Generalized correlation for gas/solid mass-transfer coefficients in metallic and ceramic foams, Industrial and Engineering Chemistry Research 46 (12) (2007) 3955-3958.

[52] H. Martin, The generalized Lévêque equation and its practical use for the prediction of heat and mass transfer rates from pressure drop, Chemical Engineering Science 57 (16) (2002) 3217-3223.

[53] J. von Rickenbach, F. Lucci, C. Narayanan, P. D. Eggenschwiler, D. Poulikakos, Effect of washcoat diffusion resistance in foam based catalytic reactors, Chemical Engineering Journal 276 (0) (2015) 388-397. doi:10.1016/j.cej.2015.03.132. 